\title{
Espace et savoirs dans Bouvard et Pécuchet
}

\section{Francis Lacoste}

\section{(2) OpenEdition}

\section{Journals}

Édition électronique

URL : http://journals.openedition.org/aes/554

DOI : $10.4000 /$ aes.554

ISSN : 2258-093X

\section{Éditeur}

Laboratoire LISAA

\section{Référence électronique}

Francis Lacoste, «Espace et savoirs dans Bouvard et Pécuchet », Arts et Savoirs [En ligne], 1 | 2012, mis en ligne le 15 février 2012, consulté le 20 avril 2019. URL : http://journals.openedition.org/aes/554 DOI : 10.4000/aes.554

Ce document a été généré automatiquement le 20 avril 2019.

Centre de recherche LISAA (Littératures SAvoirs et Arts) 


\title{
Espace et savoirs dans Bouvard et Pécuchet
}

\author{
Francis Lacoste
}

1 Bouvard et Pécuchet jouit d'un statut particulier dans la création flaubertienne, non seulement parce que l'œuvre est inachevée, mais surtout parce qu'elle instaure un nouveau rapport au savoir, en particulier aux textes écrits de la bibliothèque. Ce « livre fait de livres ", fascinant par la confrontation avec « la prolifération indéfinie du papier imprimé $»^{1}$ n'en est pas moins un roman, et même un roman d'éducation parodique, dans lequel deux retraités s'installent à la campagne afin de poursuivre une quête encyclopédique. Dès lors, on peut se demander comment les savoirs acquis sur le monde s'inscrivent dans l'espace romanesque. Or, il apparaît que Flaubert crée une nouvelle forme de roman, dans laquelle la mise en question du référent spatial laisse libre cours à l'imaginaire et oriente le texte vers une exploration intertextuelle.

2 Bouvard et Pécuchet marque une rupture avec la littérature réaliste: le référent est brouillé, et le lecteur, comme les personnages, perd ses repères. Le cadre correspondant au désir de Bouvard et Pécuchet est défini de manière très vague, voire tautologique : « Ils voulaient une campagne qui fût bien la campagne, sans tenir précisément à un site pittoresque, mais un horizon borné les attristait $»^{2}$. En fait de campagne, les deux compères vont d'ailleurs s'établir dans la Campagne de Caen, comme si l'identité du nom propre et du nom commun était garantie de ruralité. Quant au pittoresque, il ne sera certes pas excessif : «On avait en face de soi les champs, à droite une grange, avec le clocher de l'église, - et à gauche un rideau de peupliers » (66). Mais cette affligeante banalité résulte d'un choix, dicté par les idées reçues sur certaines régions et l'impossibilité d'obtenir des informations sur les autres:

Pour savoir où s'établir, ils passèrent en revue toutes les provinces. Le Nord était fertile mais trop froid, le Midi enchanteur par son climat, mais incommode vu les moustiques, et le Centre franchement n'avait rien de curieux. La Bretagne leur aurait convenu sans l'esprit cagot des habitants. Quant aux régions de l'Est, à cause $\mathrm{du}$ patois germanique, il n'y fallait pas songer. Mais il y avait d'autres pays. Qu'était-ce par exemple que le Forez, le Bugey, le Roumois? Les cartes de géographie n'en disaient rien (58). 
Cependant, les personnages se rassurent en proclamant leur indifférence au lieu, et le narrateur semble justifier leur discours par le style indirect libre, tout en ironisant car il prend ses « héros » en flagrant délit de «bourgeoisisme » : « Du reste, que leur maison fût dans tel endroit ou dans tel autre, l'important c'est qu'ils en auraient une » (58). Et cette équivalence de tous les espaces se retrouvera à la fin du roman, mais sur le mode dysphorique. Au moment où les deux amis acquièrent un esprit critique leur permettant "de voir la bêtise et de ne plus la tolérer", ils s'aperçoivent que cette bêtise est universelle : «En songeant à ce qu'on disait dans leur village, et qu'il y avait jusqu'aux antipodes d'autres Coulon, d'autres Marescot, d'autres Foureau, ils sentaient peser sur eux comme la lourdeur de toute la terre » (305).

4 Flaubert conduit ses personnages à Chavignolles au terme d'une épopée burlesque au cours de laquelle ils se perdent, puis se retrouvent de manière aléatoire, le rôle du hasard étant souligné, comme dans L'Éducation sentimentale, par la parataxe :

Cependant les ornières s'effaçaient; elles disparurent, et ils se trouvèrent au milieu des champs labourés. La nuit tombait. Que devenir? Enfin Pécuchet abandonna le chariot, et pataugeant dans la boue, s'avança devant lui à la découverte. Quand il approchait des fermes, les chiens aboyaient. Il criait de toutes ses forces pour demander sa route. On ne répondait pas. Il avait peur et regagnait le large. Tout à coup deux lanternes brillèrent. Il aperçut un cabriolet, s'élança pour le rejoindre. Bouvard était dedans.

Mais où pouvait être la voiture du déménagement? Pendant une heure, ils la hélèrent dans les ténèbres. Enfin, elle se retrouva, et ils arrivèrent à Chavignolles (64).

5 La découverte de leur maison ne donne pas lieu à une description, en raison de l'obscurité : les heureux propriétaires se contentent d'inspecter le potager à la lueur incertaine d'une bougie (65). Le lendemain, ils pourront « contempl[er]» le paysage, mais il est permis de déceler quelque ironie dans ce verbe, le jardin avec son quadrillage et son imprécision n'ayant rien d'esthétique :

Deux allées principales, formant la croix, divisaient le jardin en quatre morceaux. Les légumes étaient compris dans les plates-bandes, où se dressaient, de place en place, des cyprès nains et des quenouilles. D'un côté, une tonnelle aboutissait à un vigneau, de l'autre un mur soutenait les espaliers ; - et une claire-voie, dans le fond, donnait sur la campagne. Il y avait au-delà du mur un verger, après la charmille un bosquet, derrière la claire-voie un petit chemin. (66)

6 Cependant, ce qui surprend le plus, c'est l'absence de description de Chavignolles, le narrateur s'intéressant ensuite aux "notables", à travers le discours de la servante, comme pour suggérer que nulle part on ne peut échapper à la société, en particulier à l'ignoble bourgeoisie. La Deuxième Partie de Madame Bovary s'ouvrait sur un "morceau ", une description balzacienne de la cité, dans laquelle on pouvait entendre la voix du positiviste Homais. Rien de tel pour Chavignolles, renvoyé à son insignifiance de bourgade provinciale.

7 Mais ces effets de brouillage et d'effacement, assez caractéristiques de l'esthétique flaubertienne, ne pourraient-ils pas être mis en relation avec un thème qui revient de manière obsédante dans Bouvard et Pécuchet, celui de l'ivresse ? Lorsque Bouvard apprend qu'il hérite, il arbore le "sourire paisible des ivrognes » (58). Certes, il s'agit d'une métaphore, mais le personnage est un bon vivant, qui s'offre le plaisir de dîner une dernière fois avec son ami Barberou, si bien qu'il se trompe de diligence le lendemain, comme s'il avait l'esprit embrumé (63). Il s'intéressera particulièrement à la fabrication 
des liqueurs et créera un infect breuvage, baptisé en son honneur « la Bouvarine » (104), et il serait enclin à remplacer le culte de l'Être Suprême cher aux révolutionnaires par celui de la Nature : «Il aurait salué avec plaisir l'image d'une grosse femme, versant de ses mamelles à ses adorateurs, non pas de l'eau, mais du chambertin » (175). D'autre part, la première apparition de Gorgu revenant d'Algérie est celle d'un ivrogne : « la fatigue des bivouacs, l'absinthe et les fièvres, toute une existence de misère et de crapule se révélait dans ses yeux troubles (99). Et surtout, Flaubert étant tout sauf un naï, on peut s'interroger sur l'étrange toponyme de Chavignolles, qui combine trois sèmes éthyliques : calva + vigne + gnôle... D'ailleurs, l'alcoolisme est alors un thème à la mode (associé à une croisade vertueuse et républicaine contre le vice), et quelques années plus tard, Zola écrira L'Assommoir.

La réalité «objective» tend donc à s'effacer, si bien que la description est souvent sommaire et, contrairement à ce qui se passe souvent chez Flaubert, soumise à la diégèse. $\mathrm{Au}$ début du roman (45-47), les lieux d'où viennent les héros (la Bastille et le Jardin des Plantes) ou qu'ils aperçoivent (le Grenier d'abondance ${ }^{3}$ ) sont des amorces pour des développements à venir : délire agronomique (chapitre II), recherches sur l'histoire et en particulier sur les causes de la Révolution (chapitre IV). L'évocation de la violence révolutionnaire à travers le livre de Thiers que Pécuchet lit à haute voix, sous la tonnelle du jardin, pendant l'été 1845 , suscite en contrepoint quelques notations descriptives sur la satisfaction des personnages jouissant de la sérénité ambiante :

Des vieillards leur avaient parlé de 93 ; - et des souvenirs presque personnels animaient les plates descriptions de l'auteur. Dans ce temps-là, les grandes routes étaient couvertes de soldats qui chantaient la Marseillaise. Sur le seuil des portes, des femmes assises cousaient de la toile, pour faire des tentes. Quelquefois, arrivait un flot d'hommes en bonnet rouge, inclinant au bout d'une pique une tête décolorée, dont les cheveux pendaient. La haute tribune de la Convention dominait un nuage de poussière, où des visages furieux hurlaient des cris de mort. Quand on passait au milieu du jour près du bassin des Tuileries, on entendait le heurt de la guillotine, pareil à des coups de mouton.

Et la brise remuait les pampres de la tonnelle, les orges mûres se balançaient, par intervalles un merle sifflait. En portant des regards autour d'eux, ils savouraient cette tranquillité (175).

Jean-Pierre Richard montre bien, à travers ce texte, l'antithèse entre le passé et le présent, entre «l'humain morcelé » et «la végétalité intimisante », qui permet de mettre à distance, voire d'exorciser, le traumatisme révolutionnaire ${ }^{4}$. Mais parfois, les notations descriptives prennent une dimension bouffonne, l'hyperbole soulignant l'inanité des discours. Le débat théologique sur les martyrs, qui oppose le curé à Pécuchet un soir d'août, se situe sur fond d'orage, de véritable déluge, et le prêtre se transforme en cathédrale dégoulinante :

Le vent chassait, balayait la pluie dans l'air. Elle claquait sur les feuilles, ruisselait au bord du chemin, et le ciel couleur de boue se confondait avec les champs dénudés, la moisson étant finie. Pas un toit. Au loin seulement, la cabane d'un berger.

Le maigre paletot de Pécuchet n'avait plus un fil de sec. L'eau coulait le long de son échine, entrait dans ses bottes, dans ses oreilles, dans ses yeux, malgré la visière de la casquette Amoros. Le curé, en portant d'un bras la queue de sa soutane, se découvrait les jambes, et les pointes de son tricorne crachaient l'eau sur ses épaules comme des gargouilles de cathédrale. (337-338)

Mais ces éléments descriptifs restent limités et prennent ici une dimension burlesque. Or, on sait que beaucoup de critiques ont reproché à Flaubert d'avoir, surtout dans Salammbô et L'Éducation sentimentale, soumis la narration à la description et relégué l'homme au 
second plan. Pour Barbey d'Aurevilly, Flaubert est « un enragé descripteur », et son style, « c'est la description, une description infinie, éternelle, atomistique, aveuglante, qui tient toute la place $[. ..] \rrbracket^{5} C^{\prime}$ est un reproche qu'il ne pourra pas faire à Bouvard et Pécuchet, où la description est minimaliste, comme si Flaubert, à la fin de sa vie, entrevoyait un roman «moderne » mettant en cause l'existence de la réalité.

L'incertitude du référent laisse le champ libre à l'imaginaire. Ainsi, les toponymes ne sont plus des points de repère géographiques, mais ouvrent la voie à la rêverie de Bouvard et Pécuchet : "D'après de certains noms, ils imaginaient des pays d'autant plus beaux qu'ils n'en pouvaient rien préciser » (55). Mais cet usage " poétique » du langage n'invalide-t-il pas d'emblée la volonté de savoir encyclopédique et la philosophie positiviste des personnages?

11 Parfois, c'est l'univers intérieur qui acquiert une étonnante présence. Lassés du monde et des hommes, les deux compères s'ennuient: "Ils bâillaient l'un devant l'autre, consultaient le calendrier, regardaient la pendule, attendaient les repas ; - et l'horizon était toujours le même!» (250). Mais cet univers absurde avant la lettre peut se fracturer et prendre une profondeur bouleversante. Pécuchet surprend, dans la campagne, Madame Castillon désespérée, s'humiliant devant Gorgu qui veut l'abandonner pour aller se battre avec les républicains après le coup d'état du 2 décembre. Alors, tout un univers romantique, voire hugolien, celui de la passion, se déploie devant Pécuchet, et grâce au pouvoir de la métaphore devient réalité tangible :

Ce qu'il venait de surprendre fut pour Pécuchet comme la découverte d'un monde tout un monde ! - qui avait des lueurs éblouissantes, des floraisons désordonnées, des océans, des tempêtes, des trésors - et des abîmes d'une profondeur infinie. Un effroi s'en dégageait. Qu'importe ! Il rêva l'amour, ambitionnait de le sentir comme elle, de l'inspirer comme lui. (253)

Même le paysage le plus quotidien est vu, non comme un cadre pour la diégèse, mais comme l'expression d'un investissement affectif profond, qui exprime un rapport de l'être au monde ${ }^{6}$. Parfois, cette expérience correspond à un sentiment largement partagé, fondé sur des idées reçues: au XIX ${ }^{e}$ siècle, depuis Balzac en particulier, la campagne se caractérise par la monotonie et suscite l'ennui. Ce sentiment est suggéré dès le début de l'œuvre (" et la route, toujours la même, s'allongeait en montant jusqu'au bord de l'horizon » [62]), ce qui est de mauvais augure et relativise l'euphorie initiale lors de leur rencontre : «Comme on serait bien à la campagne! » (46). Mais l'expérience douloureuse du spleen s'exprime surtout au chapitre XII, dans un passage commenté par Jean Pierre Richard ${ }^{7}$, où le présent gnomique cache mal une confidence personnelle du Flaubert des dernières années, en proie à une " dépression mélancolique » :

Donc, ils vivaient dans cet ennui de la campagne, si lourd quand le ciel blanc écrase de sa monotonie un cœur sans espoir. On écoute le pas d'un homme en sabots qui longe le mur, ou les gouttes de la pluie tomber du toit par terre. De temps à autre, une feuille morte vient frôler la vitre, puis tournoie, s'en va. Des glas indistincts sont apportés par le vent. Au fond de l'étable, une vache mugit (250).

La description du début ( On avait en face de soi les champs, à droite une grange, avec le clocher de l'église, - et à gauche un rideau de peupliers » [66]) est d'ailleurs reprise pour souligner la permanence du paysage, mais elle acquiert une dimension subjective et suggère le caractère insupportable de la répétition et de la claustration : " - et l'horizon était toujours le même! Des champs en face, à droite l'église, à gauche un rideau de peupliers. Leurs cimes se balançaient dans la brume, perpétuellement, d'un air lamentable!» (250) 
Heureusement, il existe des moments d'euphorie où l'être, retrouvant l'homme de la nature cher à Rousseau, se perd dans la création qui offre à la fois intimité maternelle et toute puissance. Cette " effusion cosmique $»^{8}$ se donne à lire lorsque Bouvard et Pécuchet, confrontés aux insuffisances de la géologie, se laissent emporter par la rêverie «panthéistique »:

Une lisière de mousse bordait un chemin creux, ombragé par des frênes dont les cimes légères tremblaient. Des angéliques, des menthes, des lavandes exhalaient des senteurs chaudes, épicées; l'atmosphère était lourde; et Pécuchet, dans une sorte d'abrutissement, rêvait aux existences innombrables éparses autour de lui, aux insectes qui bourdonnaient, aux sources cachées sous le gazon, à la sève des plantes, aux oiseaux dans leurs nids, au vent, aux nuages, à toute la Nature, sans chercher à découvrir ses mystères, séduit par sa force, perdu dans sa grandeur. (150)

On peut toutefois se demander si l'évocation de cette rêverie de l'origine, marquée par la sensualité, n'est pas empreinte d'ironie. Dans les lignes qui suivent, la rencontre de Gorgu permet l'entrée en scène de Mélie, être de la nature, qui oriente Pécuchet vers le mystère de la femme : «Ses bandeaux, de la couleur des blés, dépassaient un béguin de toile grise. Tous ses pauvres vêtements descendaient le long de son corps sans un pli ; - et le nez droit, les yeux bleus, elle avait quelque chose de délicat, de champêtre et d'ingénu » (151). Bien entendu, il s'agit d'un piège, et Pécuchet s'apercevra à ses dépens que la fille n'est pas une ingénue...

Parfois, le désir de connaissance rationnelle et la rêverie élémentaire se concilient pour aboutir à un imaginaire scientifique tentant d'expliquer l'origine et l'avenir du monde. Un « ouvrage de la nature » assez étonnant, « une arcade qui s'ouvrait sur une grotte profonde [...] pareille à une église» (141), lance la rêverie qui, malgré l'emploi de l'indicatif, est de type pseudo-scientifique :

Bouvard penchait vers le neptunisme. Pécuchet au contraire était plutonien. Le feu central avait brisé la croûte du globe, soulevé les terrains, fait des crevasses. C'est comme une mer intérieure ayant son flux et reflux, ses tempêtes. Une mince pellicule nous en sépare. On ne dormirait pas si l'on songeait à tout ce qu'il y a sous nos talons. - Cependant le feu central diminue, et le Soleil s'affaiblit, si bien que la Terre un jour périra de refroidissement. Elle deviendra stérile ; tout le bois et toute la houille se seront convertis en acide carbonique - et aucun être ne pourra subsister. (141-142)

Excités par leur imagination délirante, les deux appentis géologues perdent conscience de la réalité, et le texte prend une dimension fantastique, non dénuée d'ironie : «Bouvard en démence, courait toujours. Le parapluie polybranches tomba, les pans de sa redingote s'envolaient, le havresac ballottait à son dos. C'était comme une tortue avec des ailes, qui aurait galopé parmi les roches. Une plus grosse le cacha. » (143-144)

Jean-Pierre Richard montre bien ce que les recherches paléontologiques des personnages (133-150) doivent au désir, et en particulier à l'inconscient. Explorer ce qui se trouve caché dans les entrailles de la terre est effraction ou infraction, et cette transgression suscite la sanction (de la part des autorités civiles, qui contrôlent les papiers, ou de la nature qui menace d'engloutir les explorateurs) ${ }^{9}$. C'est dire que le désir de connaissance (de l'espace, mais aussi du temps) est affaire de pulsion et ne peut se réduire à une exigence de rationalité. Entre l'homme et le monde, l'imaginaire et le réel, le présent et le passé, Flaubert établit un mouvement continuel qui déstabilise le lecteur mais le fait accéder à la modernité esthétique. 

Vicomte aperçu à la Vaubyessard et aspirant à la vie élégante et mystérieuse de la capitale : «Comment était-ce Paris? Quel nom démesuré ! Elle se le répétait à demi-voix, pour se faire plaisir; il sonnait à ses oreilles comme un bourdon de cathédrale, il flamboyait à ses yeux jusque sur l'étiquette de ses pots de pommade »10. La situation est inversée dans Bouvard et Pécuchet, les deux cloportes fuyant Paris pour s'installer à la campagne. On rencontre la même opposition entre le dernier roman et L'Éducation sentimentale, puisque, lorsque Frédéric se retrouve quasiment ruiné, avant d'hériter de son oncle, la routine de Nogent lui paraitt insupportable : « dans ses idées, l'art, la science et l'amour [...] dépendaient exclusivement de la Capitale $»^{11}$. D'ailleurs, les points de départ des deux romans sont symétriques: Bouvard et Pécuchet quittent Paris pour la Normandie, et à la même époque, Frédéric se rend de Paris à Nogent ${ }^{12}$. Mais les deux voyages prennent une signification opposée : les commis sont pleins d'espoir, alors que Frédéric va "languir pendant deux mois, avant d'aller faire son droit " ${ }^{13}$. On a un peu l'impression que Flaubert clôt son œuvre romanesque par une anti-Éducation sentimentale ayant pour cadre la province, en l'occurrence la Normandie.

Or, lorsque les deux compères cherchent un domaine, ils voyagent «dans tous les environs de Paris, et depuis Amiens jusqu'à Evreux, et de Fontainebleau jusqu'au Havre » (60). Si Paris et surtout Fontainebleau rappellent les amours avec Rosanette, c'est du Havre que vient Frédéric lorsqu'il rencontre Madame Arnoux sur le bateau. Et la route d'Amiens à Evreux passe par le Pays de Bray, où Bouvard et Pécuchet ont pu croiser Madame Bovary...

Mais on peut surtout être étonné par la présence de Rouen dans l'œuvre posthume. Pourquoi le galant Bouvard se trompe-t-il de diligence, se réveille-t-il «devant la cathédrale de Rouen » et se rend-il au théâtre le soir (63) ? Tous ces indices semblent être un clin d'œil à Madame Bovary: il est vrai que, comme on l'a souvent dit, les patronymes de Bouvard et de Bovary sont étonnamment proches.

Plus inattendue est la référence implicite à Salammbô, en particulier à cette "pointe d'imagination sadique » décelée par Sainte-Beuve ${ }^{14}$ et qui s'exprime en particulier lors du sacrifice des enfants. Or, l'excursion de Bouvard et Pécuchet au menhir de Passais aboutit à la contemplation de masses de granit :

La plus considérable est creusée comme un bassin. Un des bords se relève - et du fond partent deux entailles qui descendent jusqu'à terre. C'était pour l'écoulement du sang; impossible d'en douter ! Le hasard ne fait pas de ces choses.

Les racines des arbres s'entremêlaient à ces rocs abrupts. Un peu de pluie tombait ; au loin, les flocons de brume montaient, comme de grands fantômes. Il était facile d'imaginer sous les feuillages, les prêtres en tiare d'or et en robe blanche, avec leurs victimes humaines les bras attachés dans le dos - et sur le bord de la cuve la druidesse, observant le ruisseau rouge, pendant qu'autour d'elle, la foule hurlait, au tapage des cymbales et des buccins faits d'une corne d'auroch. (166-167)

De même que, dans Salammbô, le fait qu'il ne reste rien de Carthage permettait à Flaubert de laisser libre cours à des fantasmes inspirés par le divin Marquis, de même ici, l'absence de certitude historique - puisque du monde des druides, "il ne reste que des pierres » 
(166) - autorise les deux compères à imaginer les scènes les plus atroces de sacrifice humain.

Mais l'intertextualité est également très présente, si bien que Bouvard et Pécuchet nous promènent à travers la littérature. Les provinces françaises sont liées à des stéréotypes idéologiques associés à des œuvres. Ainsi «l'esprit cagot des habitants » de la Bretagne (59) fait-il écho aux Chouans de Balzac, mais surtout à Quatre-vingt-Treize, publié par Hugo en 1874. Et dans la phrase suivante, le rejet des provinces de l'Est, à cause du patois germanique, s'explique par l'actualité au moment de l'écriture (la perte de l'AlsaceLorraine) mais pourrait aussi rappeler la vogue des Romans nationaux d'ErckmannChatrian, que Flaubert méprisait.

Les promenades dans la campagne autour de Paris, avant l'héritage, sont marquées par la philosophie rousseauiste de la nature, mais la retombée finale souligne la naïveté des personnages et dénonce les clichés sur la campagne :

Un dimanche ils se mirent en marche dès le matin; et passant par Meudon, Bellevue, Suresnes, Auteuil, tout le long du jour ils vagabondèrent entre les vignes, arrachèrent des coquelicots au bord des champs, dormirent sur l'herbe, burent du lait, mangèrent sous les acacias des guinguettes, et rentrèrent fort tard, poudreux, exténués, ravis. Ils renouvelèrent souvent ces promenades. Les lendemains étaient si tristes qu'ils finirent par s'en priver (55).

Et au-delà de l'idylle rousseauiste ou sandienne, c'est le roman rustique, très à la mode dans la deuxième moitié du XIX ${ }^{e}$ siècle ${ }^{15}$ qui semble visé. Le rêve de Bouvard et Pécuchet permet d'ironiser sur une littérature qui exalte une économie d'autosuffisance :

Déjà, ils se voyaient en manches de chemise, au bord d'une plate-bande émondant des rosiers, et bêchant, binant, maniant de la terre, dépotant des tulipes. Ils se réveilleraient au chant de l'alouette, pour suivre les charrues, iraient avec un panier cueillir des pommes, regarderaient faire le beurre, battre le grain, tondre les moutons, soigner les ruches, et se délecteraient au mugissement des vaches et à la senteur des foins coupés. Plus d'écritures! Plus de chefs! Plus même de terme à payer ! - Car ils posséderaient un domicile à eux! Et ils mangeraient les poules de leur basse-cour, les légumes de leur jardin, et dîneraient en gardant leurs sabots ! « Nous ferons tout ce qui nous plaira! Nous laisserons pousser notre barbe ! (59)

La réalité ne tardera pas à dénoncer cette utopie; Bouvard et Pécuchet, confrontés à la cupidité et à la malhonnêteté des paysans ${ }^{16}$, se ruineront, et ce «couple» de bourgeois retraités qui s'installe à la campagne donne une image parodique du retour à la terre ${ }^{17}$ et à la nature. Quant au rêve agronomique de Bouvard, il n'aboutit pas à une exigence de pureté, mais à l'amalgame organique de la fosse aux composts, qui ramène à la réalité de la vie paysanne :

Excité par Pécuchet, il eut le délire de l'engrais. Dans la fosse aux composts furent entassés des branchages, du sang, des boyaux, des plumes, tout ce qu'il pouvait découvrir. Il employa la liqueur belge, le lisier suisse, la lessive $\mathrm{Da}$-Olmi, des harengs saurs, du varech, des chiffons, fit venir du guano, tâcha d'en fabriquer - et poussant jusqu'au bout ses principes, ne tolérait pas qu'on perdît l'urine; il supprima les lieux d'aisances. On apportait dans sa cour des cadavres d'animaux, dont il fumait ses terres. Leurs charognes dépecées parsemaient la campagne. Bouvard souriait au milieu de cette infection. Une pompe installée dans un tombereau crachait du purin sur les récoltes. À ceux qui avaient l'air dégoûté, il disait : « Mais c'est de l'or ! C'est de l'or. » - Et il regrettait de n'avoir pas encore plus de fumiers. Heureux les pays où l'on trouve des grottes naturelles pleines d'excréments d'oiseaux ! (81)

Ici, on n'est pas très loin de Zola, et on comprend que Barbey ${ }^{18}$, avec l'outrance qui le caractérise, fasse de Flaubert un naturaliste. 


\section{grand artiste, l'auteur des Fleurs du Mal :}

Ils voulurent faire comme autrefois une promenade dans les champs, allèrent très loin, se perdirent. - De petits nuages moutonnaient dans le ciel, le vent balançait les clochettes des avoines, le long d'un pré un ruisseau murmurait, quand tout à coup une odeur infecte les arrêta. Et ils virent sur des cailloux, entre des ronces, la charogne d'un chien.

Les quatre membres étaient desséchés. Le rictus de la gueule découvrait sous des babines bleuâtres des crocs d'ivoire. A la place du ventre, c'était un amas de couleur terreuse, et qui semblait palpiter tant grouillait dessus la vermine. Elle s'agitait, frappée par le soleil, sous le bourdonnement des mouches, dans cette intolérable odeur, une odeur féroce et comme dévorante. (307)

Mais ici, la charogne grouillant de vers n'est pas transfigurée par l'art, et l'odeur « féroce et comme dévorante" conduit à une réflexion philosophique sur la mort puis à la tentation du suicide - qui se soldera par un ratage, comme tout ce que les deux amis entreprennent.

L'art serait-il donc incapable de conjurer l'angoisse existentielle ? En tout cas, les velléités des deux esthètes amateurs aboutissent à une monstruosité et condensent les aberrations esthétiques. Inspirés par l'ouvrage de Boitard L'Architecte des jardins, Bouvard et Pécuchet doivent choisir entre différents styles, ou plutôt entre divers clichés dénoncés par l'ironie du narrateur :

Il y a, d'abord, le genre mélancolique et romantique, qui se signale par des immortelles, des ruines, des tombeaux, et « un ex-voto à la Vierge, indiquant la place où un seigneur est tombé sous le fer d'un assassin». On compose le genre terrible avec des rocs suspendus, des arbres fracassés, des cabanes incendiées, le genre exotique en plantant des cierges du Pérou « pour faire naître des souvenirs à un colon ou à un voyageur ». Le genre grave doit offrir, comme Ermenonville, un temple à la philosophie. Les obélisques et les arcs de triomphe caractérisent le genre majestueux, de la mousse et des grottes le genre mystérieux, un lac le genre rêveur. Il y a même le genre fantastique, dont le plus beau spécimen se voyait naguère dans un jardin wurtembergeois - car, on y rencontrait successivement, un sanglier, un ermite, plusieurs sépulcres, et une barque se détachant d'elle-même du rivage, pour vous conduire dans un boudoir, où des jets d'eau vous inondaient, quand on se posait sur le sofa. (91-92)

Ce réjouissant bric-à-brac associe le tombeau de Rousseau à Ermenonville, les grottes d'Ossian et l'inévitable lac de Lamartine, tout en déclinant les thèmes éculés de la littérature romantique : esthétique des ruines, obsession de la mort, épopée, religiosité, exotisme et fantastique/merveilleux. Au terme de leurs travaux, Bouvard et Pécuchet proposent aux notables qu'ils ont réunis un chef-d'œuvre kitsch qui n'a rien à envier à la casquette de Charles et qui suscite les sarcasmes du narrateur :

C'était dans le crépuscule, quelque chose d'effrayant. Le rocher comme une montagne occupait le gazon, le tombeau faisait un cube au milieu des épinards, le pont vénitien un accent circonflexe par-dessus les haricots - et la cabane, au-delà, une grande tache noire; car ils avaient incendié son toit, pour la rendre plus poétique. Les ifs en forme de cerfs ou de fauteuils se suivaient, jusqu'à l'arbre foudroyé, qui s'étendait transversalement de la charmille à la tonnelle, où des pommes d'amour pendaient comme des stalactites. Un tournesol, çà et là, étalait son disque jaune. La pagode chinoise peinte en rouge semblait un phare sur le vigneau. Les becs des paons frappés par le soleil se renvoyaient des feux, et derrière la claire-voie, débarrassée de ses planches, la campagne toute plate terminait l'horizon. (97-98) 
Si le résultat est « effrayant ", c'est sans doute parce que les deux bourgeois manquent de goût, mais surtout parce qu'ils ont la prétention de modeler la nature en fonction de clichés pour en faire une œuvre d'art. Le véritable artiste - en l'occurrence Flaubertserait donc celui qui voyage à travers la littérature en adoptant un point de vue critique, voire ironique.

Perdus dans un espace indéfini et insignifiant, sur « un plateau stupide, entre Caen et Falaise $»^{19}$, Bouvard et Pécuchet voient la réalité se troubler ou se dérober, ce qui désoriente le lecteur. Il ne s'agit donc pas de donner «l'illusion du réel ", de situer l'intrigue dans un cadre auquel la description fournira une vraisemblance. Au contraire, l'espace créé par la diégèse et modelé par l'imaginaire devient le lieu de la subjectivité, de la rêverie, et la crise de la représentation aboutit à une vision poétique du monde qui met en question la littérature "réaliste ». Mais dans une démarche autotélique et circulaire, Flaubert, à travers son "encyclopédie critique en farce ${ }^{20}$ et son Dictionnaire des idées reçues, rend problématique la possibilité de la connaissance et dénonce par l'ironie les stéréotypes idéologiques et littéraires, autrement dit la Bêtise omniprésente. Roman laborieux, par lequel l'auteur se dit «abruti », Bouvard et Pécuchet manifeste à la fois l'amour et la défiance envers la littérature.

\section{NOTES}

1. Michel Foucault, "La bibliothèque fantastique », Travail de Flaubert, Éditions du Seuil, coll. Points Essais, 1983, p. 118.

2. Flaubert, Bouvard et Pécuchet (édition de référence), GF Flammarion, 2011 (édition de Stéphanie Dord-Crouslé), p. 60.

3. Le Grenier d'abondance porte doublement la marque de l'histoire, car il fut créé par Napoléon en 1807 et incendié en 1871 lors de la Commune. Juste après cette mention, les deux compères entament « une conversation politique » sur les ouvriers.

4. Jean-Pierre Richard, « Variation d'un paysage », Travail de Flaubert, op.cit., p. 190-192.

5. Jules Barbey d'Aurevilly, « L'Éducation sentimentale. Histoire d'un jeune homme par M. Gustave Flaubert », Le Constitutionnel, 29 novembre 1869 (voir Didier Philippot, Gustave Flaubert, Mémoire de la critique, PUPS, 2006, p. 297).

6. Voir Jean-Pierre Richard, op. cit., p. 179-197.

7. Ibid., p. 187-189.

8. Ibid., p. 193.

9. Ibid., p. 193-197.

10. Flaubert, Madame Bovary, Classiques de Poche, 2005, p. 127.

11. Flaubert, L'Éducation sentimentale, GF Flammarion, 1985, p. 145.

12. L'Éducation sentimentale commence le 15 septembre 1840, et Pécuchet part de Paris le 20 mars 1841.

13. Ibid., p. 47.

14. Sainte-Beuve, «Salammbô, par M. Gustave Flaubert », Le Constitutionnel, 8, 15 et 22 décembre 1862 (voir Didier Philippot, op.cit., p. 217).

15. Voir Paul Vernois, Le Roman rustique de George Sand à Ramuz, Nizet, 1962. 
16. «Les gens de la campagne, meilleurs que ceux des villes; envier leur sort » dit le Dictionnaire des idées_reçues, p. 421.

17. Le thème du retour à la terre, illustré dans le roman « rustique social » d'Eugène Le Roy par exemple, servira la politique protectionniste de Méline à la fin du siècle.

18. «[...] la haine du bourgeois, dans Flaubert, va jusqu'à cette fange qu'il remue, et qu'il remue, en naturaliste, sans indignation, sans dégoût, sans nausée, avec l'impassibilité d'un homme qui a perdu la délicatesse de l'artiste » («Bouvard et Pécuchet, par Gustave Flaubert », Le Constitutionnel, 10 mai 1881. Voir Didier Philippot, op.cit., p. 529).

19. Flaubert, Correspondance, tome IV, Gallimard, Bibliothèque de la Pléiade, 1997, p. 816 (lettre du 24 juin 1874).

20. Ibid., p. 559 (lettre à Edma Roger des Genettes du 19 août 1872).

INDEX

Mots-clés : savoir, intertextualité, Éducation sentimentale (L'), Madame Bovary, Salammbô, Dictionnaire des idées reçues

\section{AUTEUR}

\section{FRANCIS LACOSTE}

ISEAH de Tozeur, Tunisie 